\title{
Weighted Sum Rate Optimization for STAR-RIS-assisted MIMO System
}

\author{
Hehao Niu, Zheng Chu, Member, IEEE, Fuhui Zhou, Senior Member, IEEE, \\ Pei Xiao, Senior Member, IEEE, and Naofal Al-Dhahir, Fellow, IEEE
}

\begin{abstract}
In this work, we study a simultaneous transmitting and reflecting reconfigurable intelligent surface (RIS)-assisted multiple-input multiple-output network. For the system under consideration, we maximize the weighted sum rate, mainly based on the energy splitting (ES) scheme. To tackle this optimization problem, a sub-optimal block coordinate descent (BCD) algorithm is proposed to design the precoding matrices and the transmitting and reflecting coefficients (TRCs) in an alternate manner. Specifically, the precoding matrices are solved using the Lagrange dual method, while the TRCs are obtained using the constrained concave-convex procedure (CCCP). The simulation results reveal that: 1) Simultaneous transmitting and reflecting RIS (STAR-RIS) can achieve better performance than conventional reflecting/transmiting-only RIS; 2) In unicast communication, time switching (TS) scheme outperforms the ES and mode selection (MS) schemes, while in broadcast communication, ES scheme outperforms the TS and MS schemes.
\end{abstract}

Index Terms-Reconfigurable intelligent surfaces, simultaneously transmitting and reflecting, block coordinate descent, constrained concave-convex procedure.

\section{INTRODUCTION}

Nowadays, the reconfigurable intelligent surface (RIS) has attracted great interest from both industry and academia. Since RIS can be installed on flat surfaces to reflect wireless signals and establish a virtual end-to-end link between the transmitter (Tx) and the receiver (Rx), RIS has emerged as a key technology in future wireless systems [1]. Recently, RIS has sparked significant works in various wireless applications such as the multiple-input multiple-output (MIMO) networks [2], energy harvesting network [3], and unmanned aerial vehicle (UAV) networks [4], among others.

However, most existing works consider the reflecting-only RIS, e.g., the RIS can only reflect the incident signal. As such, Tx and Rx need to be located on the same side of the RIS, which restricts the deployment of RIS [5]. To overcome

This work was supported in part by the National Natural Science Foundation of China under Grant 61901490, 62071223, and 62031012, in part by the U.K. Engineering and Physical Sciences Research Council under Grant $\mathrm{EP} / \mathrm{P} 03456 \mathrm{X} / 1$.

H. Niu is with the Institute of Electronic Countermeasure, National University of Defense Technology, Hefei, 230037, China, (e-mail: niuhaonup$\mathrm{t} @$ foxmail.com).

Z. Chu and P. Xiao are with 5GIC \& 6GIC, Institute for Communication Systems (ICS), University of Surrey, Guildford GU2 7XH, United Kingdom. (Email: andrew.chuzheng7@gmail.com,p.xiao@surrey.ac.uk)

F. Zhou is with the College of Electronic and Information Engineering, Nanjing University of Aeronautics and Astronautics, Nanjing, 210000, China. (e-mail: zhoufuhui@ieee.org).

N. Al-Dhahir is with the Department of Electrical and Computer Engineering, The University of Texas at Dallas, Richardson, TX 75080 USA. (email:aldhahir@utdallas.edu). this shortcoming, recently, a novel type of RIS, named simultaneous transmitting and reflecting RIS (STAR-RIS) [6], or intelligent omni-surface [7], was proposed. Different from reflecting-only RIS, STAR-RIS can transmit and reflect the signal simultaneous, thus to obtain a full-space coverage [8]. By adjusting the electromagnetic characteristics of the elements, the transmitting and reflecting coefficients (TRCs) can be designed to control the transmitted and reflected signals [9]. Currently, to achieve independent control of both the transmitted and reflected signals, in lower frequency $(10 \mathrm{KHz}$ up to $1 \mathrm{GHz}$ ), STAR-RIS is commonly implemented by patcharray, while in higher frequency, it is commonly implemented by graphene based meta-materials, respectively [10]. Then, three protocols, e.g., energy splitting (ES), mode selection (MS), and time switching (TS), were proposed to operate the STAR-RIS in a multiple-input single-output (MISO) networks [11]. However, the synergy between the transmission (T) mode and the reflection $(\mathrm{R})$ mode in STAR-RIS-assisted MIMO network has not been studied yet.

Against this background, this work investigates the joint precoding matrices and TRCs optimization in STAR-RIS-aided MIMO networks. Firstly, we utilize an equivalent method to reformulate the weighted sum rate (WSR) objective. Then, the precoding matrices are found by the Lagrange dual method, while the TRCs are obtained by the constrained concaveconvex procedure (CCCP). The simulation results revealed the superiority of the proposed approach and indicated that: 1) STAR-RIS outperforms the reflecting-only RIS; 2) The TS scheme is more beneficial to unicast communication; 3) The ES scheme is a better choice for broadcast communication.

The main difference between our work and the most related work [11] are summarized as follows: 1) For the system model and problem formulation, we investigate the STAR-RISassisted MIMO network to maximize the WSR. While [11] considered the power minimization design in a STAR-RISassisted MISO network; 2) Regarding the used optimization method, [11] mainly used the semidefinite relaxation (SDR) and successive inner-approximation, while our method is mainly based on the block coordinate descent (BCD) method, Lagrange dual, and CCCP, which leads to better performance than the SDR method.

\section{Signal Model And Protocols of STAR-RIS}

This part provides the signal model and operating protocols of STAR-RIS.

\section{A. Signal Model}

Assume that the STAR-RIS has $M$ elements and $s_{m}$ denotes the incident signal to the $m$-th element, $m \in \mathcal{M} \triangleq$ $\{1,2, \ldots, M\}$, then the transmitted and reflected signals by 
the $m$-th element can be denoted as $t_{m}=\sqrt{\alpha_{m}^{t}} e^{j \phi_{m}^{t}} s_{m}$, and $r_{m}=\sqrt{\alpha_{m}^{r}} e^{j \phi_{m}^{r}} s_{m}$, where $\sqrt{\alpha_{m}^{t}} \in[0,1], \phi_{m}^{t} \in[0,2 \pi)$ and $\sqrt{\alpha_{m}^{r}} \in[0,1], \phi_{m}^{r} \in[0,2 \pi)$ are the amplitude and phase responses of the $m$-th element, respectively. Then, according to [9], when the surface electric and magnetic impedance for a STAR-RIS is adjustable, $\phi_{m}^{t}$ and $\phi_{m}^{r}$ can be chosen independently. However, $\sqrt{\alpha_{m}^{t}}$ and $\sqrt{\alpha_{m}^{r}}$ need to satisfy the energy conservation constraint $\alpha_{m}^{t}+\alpha_{m}^{r}=1, \forall m \in \mathcal{M}$.

\section{B. Three Operating Protocols}

Here, we provide the main points of the three protocols. More details are discussed in [11].

1) ES protocol, where all elements simultaneously operate in the $\mathrm{T}$ and $\mathrm{R}$ modes. Then, the TRCs are expressed as $\boldsymbol{\Phi}_{t}^{E S}=\operatorname{Diag}\left(\sqrt{\alpha_{1}^{t}} e^{j \phi_{1}^{t}}, \ldots, \sqrt{\alpha_{M}^{t}} e^{j \phi_{M}^{t}}\right)$, and $\boldsymbol{\Phi}_{r}^{E S}=$ $\operatorname{Diag}\left(\sqrt{\alpha_{1}^{r}} e^{j \phi_{1}^{r}}, \ldots, \sqrt{\alpha_{M}^{r}} e^{j \phi_{M}^{r}}\right)$, where $\alpha_{m}^{t}, \alpha_{m}^{r} \in[0,1]$, $\alpha_{m}^{t}+\alpha_{m}^{r}=1$, and $\phi_{m}^{t}, \phi_{m}^{r} \in[0,2 \pi), \forall m \in \mathcal{M}$.

2) MS protocol, where all elements are divided into two groups with $M_{t}$ and $M_{r}$ elements, respectively. Then, one group operating in the T mode, and the other group operating in the $\mathrm{R}$ mode. Here, $M_{t}+M_{r}=M$. Then, the TRCs are expressed as $\boldsymbol{\Phi}_{t}^{M S}=\operatorname{Diag}\left(\sqrt{\alpha_{1}^{t}} e^{j \phi_{1}^{t}}, \ldots, \sqrt{\alpha_{M}^{t}} e^{j \phi_{M}^{t}}\right)$, and $\boldsymbol{\Phi}_{r}^{M S}=\operatorname{Diag}\left(\sqrt{\alpha_{1}^{r}} e^{j \phi_{1}^{r}}, \ldots, \sqrt{\alpha_{M}^{r}} e^{j} \phi_{M}^{r}\right)$, respectively, where $\alpha_{m}^{t}, \alpha_{m}^{r} \in\{0,1\}, \alpha_{m}^{t}+\alpha_{m}^{r}=1$, and $\phi_{m}^{t}, \phi_{m}^{r} \in$ $[0,2 \pi), \forall m \in \mathcal{M}$.

3) TS protocol, where all elements are switched in orthogonal time slots between the two modes. Here, the slot lengths to the $\mathrm{T}$ and $\mathrm{R}$ modes are denoted as $\tau_{t}$ and $\tau_{r}$, respectively, with $\tau_{t}+\tau_{r}=1$. Then, the TRCs are expressed as $\boldsymbol{\Phi}_{t}^{T S}=\operatorname{Diag}\left(e^{j \phi_{1}^{t}}, e^{j \phi_{2}^{t}}, \ldots, e^{j \phi_{M}^{t}}\right)$ and $\boldsymbol{\Phi}_{r}^{T S}=$ $\operatorname{Diag}\left(e^{j \phi_{1}^{r}}, e^{j \phi_{2}^{r}}, \ldots, e^{j \phi_{M}^{r}}\right)$, respectively, where $\phi_{m}^{t}, \phi_{m}^{r} \in$ $[0,2 \pi), \forall m \in \mathcal{M}$.

\section{System Model AND PROBlem Formulation}

In the section, a MIMO system assisted by a STAR-RIS is considered first. Then, the WSR objective is formulated for these protocols.

\section{A. System Model}

A STAR-RIS assisted MIMO system is investigated, where a Tx intends to communicate with two users with the assistance of a $M$ element STAR-RIS. We assume that the RIS can converge the $\mathrm{T}$ and $\mathrm{R}$ regions simultaneously [11]. To be specific, the user deployed in the $\mathrm{T}$ region is denoted as the $\mathrm{T}$ user, while the other one in the $\mathrm{R}$ region is known as the $\mathrm{R}$ user. Let $\mathbf{F} \in \mathbb{C}^{M \times N}, \mathbf{H}_{t} \in \mathbb{C}^{N_{t} \times M}$, and $\mathbf{H}_{r} \in \mathbb{C}^{N_{r} \times M}$ denote the channel matrices between Tx and RIS, RIS and the T user, RIS and the R user, respectively, where $N, N_{t}$ and $N_{r}$ are the numbers of antennas for Tx, the $\mathrm{T}$ user and the $\mathrm{R}$ user, respectively. Here, we assume that perfect channel state information (CSI) of these links are obtained by Tx, and the direct links from Tx to the users are blocked. In addition, a field-programmable gate array enabled controller is used to operate the RIS.

Let $\mathbf{s}_{l} \in \mathbb{C}^{N_{d_{l}} \times 1}, l \in\{t, r\}$ be the transmit symbols for the $l$-th user with $N_{d_{l}}$ being the corresponding number of data streams. Thus, when the ES or MS scheme is used, the signal received by the $l$-th user is given as

$$
\mathbf{y}_{l}=\mathbf{H}_{l} \boldsymbol{\Phi}_{l} \mathbf{F} \sum_{l} \mathbf{W}_{l} \mathbf{s}_{l}+\mathbf{n}_{l}
$$

where $\mathbf{W}_{l} \in \mathbb{C}^{N \times N_{d_{l}}}$ denotes the precoding matrix for the $l$-th user, $\boldsymbol{\Phi}_{l} \in \mathbb{C}^{M \times M}$ is the STAR-RIS coefficient matrix, $\mathbf{n}_{l} \in \mathbb{C}^{N_{l} \times 1}$ is the noise vector at the $l$-th user with $\mathbf{n}_{l} \sim \mathcal{C N}\left(\mathbf{0}, \sigma_{l}^{2} \mathbf{I}\right)$, and $\sigma_{l}^{2}$ is the noise power. Here, we assume that $\mathbf{s}_{t}$ and $\mathbf{s}_{r}$ are independent, since we study unicast communication. On the other hand, for broadcast communication, the Tx sends the same information to the users. In the following, we mainly focus on the unicast scenario. However, the proposed method can be extended the broadcast scenario.

By denoting $\overline{\mathbf{H}}_{l}=\mathbf{H}_{l} \boldsymbol{\Phi}_{l} \mathbf{F} / \sigma_{l}$, the achievable information rate for the $l$-th user is given by

$$
R_{l}=\ln \left|\mathbf{I}+\overline{\mathbf{H}}_{l} \mathbf{W}_{l} \mathbf{W}_{l}^{H} \overline{\mathbf{H}}_{l}^{H} \mathbf{C}_{l}^{-1}\right|,
$$

where $\mathbf{C}_{l}=\overline{\mathbf{H}}_{l} \mathbf{W}_{l^{\prime}} \mathbf{W}_{l^{\prime}}^{H} \overline{\mathbf{H}}_{l}^{H}+\mathbf{I}$ is the interference plus noise covariance matrix. When $l=r, l^{\prime}=t$, and vice versa.

While for the TS scheme, since different users are allocated different time slots, the information rate for the $l$-th user is given by

$$
R_{l}=\tau_{l} \ln \left|\mathbf{I}+\overline{\mathbf{H}}_{l} \mathbf{W}_{l} \mathbf{W}_{l}^{H} \overline{\mathbf{H}}_{l}^{H}\right|
$$

\section{B. Optimization Problem Formulation}

We aim to maximize the WSR by optimizing the precoding matrices and the TRCs. Specifically, when the ES scheme is used, the problem is given as:

$$
\begin{aligned}
\text { ES : } & \max _{\mathbf{W}_{l}, \mathbf{\Phi}_{l}} \sum_{l} \omega_{l} R_{l} \\
\text { s.t. } & {\left[\mathbf{\Phi}_{l}\right]_{m}=\sqrt{\alpha_{m}^{l}} e^{j \phi_{m}^{l}}, \alpha_{m}^{l} \in[0,1], \phi_{m}^{l} \in[0,2 \pi), } \\
& \sum_{l} \operatorname{Tr}\left(\mathbf{W}_{l} \mathbf{W}_{l}^{H}\right) \leq P_{s},
\end{aligned}
$$

where $\omega_{l} \in[0,1], \sum_{l} \omega_{l}=1$ is the weight for the $l$-th user, and $P_{s}$ denotes the maximum transmit power. In fact, by replacing $\alpha_{m}^{l} \in[0,1]$ with $\alpha_{m}^{l} \in\{0,1\}$, (4) can be applied to the MS scheme.

Then, when the TS scheme is utilized, the WSR problem is formulated as

$$
\begin{array}{ll}
\text { TS : } & \max _{\mathbf{W}_{l}, \mathbf{\Phi}_{l}, \tau_{l}} \sum_{l} w_{l} \tau_{l} R_{l} \\
\text { s.t. } & {\left[\boldsymbol{\Phi}_{l}\right]_{m}=e^{j \phi_{m}^{l}}, \phi_{m}^{l} \in[0,2 \pi),} \\
& \tau^{l} \in[0,1], \sum_{l} \tau^{l}=1, \\
& \sum_{l} \tau_{l} \operatorname{Tr}\left(\mathbf{W}_{l} \mathbf{W}_{l}^{H}\right) \leq P_{s} .
\end{array}
$$

In fact, for reflecting-only RIS, the main constraint is the unit modulus constraint, and several effective approaches such as manifold optimization (MO) [2], and MajorizationMinimization (MM) [3], were proposed to handle the problem. While for STAR-RIS, the coefficients for the T and R modes are coupled by the energy conservation constraint. Thus, the optimization of STAR-RIS is different from that for reflectingonly RIS. In the following section, we will develop an effective method to solve (4).

\section{JOINT PRECODING AND TRCS DESIGN}

In this section, we focus on the precoding matrices and TRCs design for the ES scheme, where we transform the intractable objective function (OF) (4a) into an equivalent form. Then, a BCD-based method is proposed to solve it. ${ }^{1}$

\footnotetext{
${ }^{1}$ Here, we mainly focus on the ES scheme. In fact, the method proposed for the ES scheme can be extended to the MS scheme by using the approach in [12, Section V]. As for the TS scheme, since the T and R coefficients are independent, the proposed framework in [2] or [3] can be utilized to optimize the precoding matrices and the phase shifters. Then, a one-dimensional search technique can be utilized to determine the optimal slot lengths $\tau_{t}$ and $\tau_{r}$. We omit the details due to space limitations.
} 


\section{A. Reformulation of Problem (4)}

The following Lemma is useful to handle (4).

Lemma 1 [13]: Define a matrix function

$\mathbf{Z}(\mathbf{U}, \mathbf{W}) \triangleq\left(\mathbf{I}-\mathbf{U}^{H} \mathbf{M W}\right)\left(\mathbf{I}-\mathbf{U}^{H} \mathbf{M} \mathbf{W}\right)^{H}+\mathbf{U}^{H} \mathbf{N U}$, where $\mathbf{N} \succ \mathbf{0}$. Then, the following equations hold true.

1) For any $\mathbf{Z} \in \mathbb{C}^{m \times m}$ satisfying $\mathbf{Z} \succ \mathbf{0}$, we have

$$
\mathbf{Z}^{-1}=\underset{\mathbf{\Psi} \succ \mathbf{0}}{\arg \max } \ln |\mathbf{\Psi}|-\operatorname{Tr}(\mathbf{\Psi} \mathbf{Z})
$$

and

$$
-\ln |\mathbf{Z}|=\underset{\mathbf{\Psi} \succ \mathbf{0}}{\arg \max } \ln |\mathbf{\Psi}|-\operatorname{Tr}(\mathbf{\Psi} \mathbf{Z})+m .
$$

2) For any $\boldsymbol{\Psi} \succ \mathbf{0}$, we have

$$
\begin{aligned}
\tilde{\mathbf{U}} & \triangleq \underset{\mathbf{U}}{\arg \min } \operatorname{Tr}(\mathbf{\Psi} \mathbf{Z}(\mathbf{U}, \mathbf{W})) \\
& =\left(\mathbf{N}+\mathbf{M} \mathbf{W} \mathbf{W}^{H} \mathbf{M}^{H}\right)^{-1} \mathbf{M W},
\end{aligned}
$$

$\mathbf{Z}(\tilde{\mathbf{U}}, \mathbf{W})=\mathbf{I}-\tilde{\mathbf{U}}^{H} \mathbf{M} \mathbf{W}=\left(\mathbf{I}+\mathbf{W}^{H} \mathbf{M}^{H} \mathbf{N}^{-1} \mathbf{M} \mathbf{W}\right)^{-1}$.

3) We have

$$
\begin{aligned}
& \ln \left|\mathbf{I}+\mathbf{M} \mathbf{W} \mathbf{W}^{H} \mathbf{M}^{H} \mathbf{N}^{-1}\right|= \\
& \max _{\mathbf{\Psi} \mathbf{0}, \mathbf{U}} \ln |\mathbf{\Psi}|-\operatorname{Tr}(\mathbf{\Psi} \mathbf{Z}(\mathbf{U}, \mathbf{W}))+m .
\end{aligned}
$$

Then, we derive an equivalent reformulation of (4) by Lemma 1. Firstly, we define

$$
\begin{array}{r}
\mathbf{Z}_{l}\left(\mathbf{U}_{l},\left\{\mathbf{W}_{l}, \boldsymbol{\Phi}_{l}\right\}\right) \triangleq \mathbf{U}_{l}^{H}\left(\mathbf{I}+\overline{\mathbf{H}}_{l} \mathbf{W}_{l^{\prime}} \mathbf{W}_{l^{\prime}}^{H} \overline{\mathbf{H}}_{l}^{H}\right) \mathbf{U}_{l} \\
+\left(\mathbf{I}-\mathbf{U}_{l}^{H} \overline{\mathbf{H}}_{l} \mathbf{W}_{l}\right)\left(\mathbf{I}-\mathbf{U}_{l}^{H} \overline{\mathbf{H}}_{l} \mathbf{W}_{l}\right)^{H} .
\end{array}
$$

Then, by Equation 3, we obtain

$$
\begin{aligned}
& \ln \left|\mathbf{I}+\overline{\mathbf{H}}_{l} \mathbf{W}_{l} \mathbf{W}_{l}^{H} \overline{\mathbf{H}}_{l}^{H} \mathbf{C}_{l}^{-1}\right|= \\
& \max _{\mathbf{\Psi}_{l} \succ \mathbf{0}, \mathbf{U}_{l}} \ln \left|\mathbf{\Psi}_{l}\right|-\operatorname{Tr}\left(\boldsymbol{\Psi}_{l} \mathbf{Z}_{l}\left(\mathbf{U}_{l},\left\{\mathbf{W}_{l}, \boldsymbol{\Phi}_{l}\right\}\right)\right)+d_{l} .
\end{aligned}
$$

Thus, by substituting (7) into (4), (4) can be equivalently reformulated as

$$
\max _{\substack{\mathbf{W}_{l}, \Phi_{l}, \mathbf{U}_{l}, \Psi_{l}}} \sum_{l} \omega_{l}\left(\ln \left|\Psi_{l}\right|-\operatorname{Tr}\left(\mathbf{\Psi}_{l} \mathbf{Z}_{l}\left(\mathbf{U}_{l},\left\{\mathbf{W}_{l}, \mathbf{\Phi}_{l}\right\}\right)\right)\right)
$$

s.t. $(4 \mathrm{~b}),(4 \mathrm{c}), \boldsymbol{\Psi}_{l} \succ \mathbf{0}$.

Next, we utilize the BCD method to handle (8), where the whole procedure includes the following sub-iterations.

1) Step 1: Solve (8) for $\mathbf{U}_{l}$ while fixing $\left\{\mathbf{W}_{l}, \boldsymbol{\Phi}_{l}, \boldsymbol{\Psi}_{l}\right\}$, which is equivalent to minimizing $\operatorname{Tr}\left(\mathbf{\Psi}_{l} \mathbf{Z}_{l}\left(\mathbf{U}_{l},\left\{\mathbf{W}_{l}, \mathbf{\Phi}_{l}\right\}\right)\right)$ over $\mathbf{U}_{l}$. According to Equation (2), the optimal $\mathbf{U}_{l}$ is:

$$
\mathbf{U}_{l}=\left(\overline{\mathbf{H}}_{l} \mathbf{W}_{l} \mathbf{W}_{l}^{H} \overline{\mathbf{H}}_{l}^{H}+\mathbf{C}_{l}\right)^{-1} \overline{\mathbf{H}}_{l} \mathbf{W}_{l} \text {. }
$$

2) Step 2: Solve (8) for $\boldsymbol{\Psi}_{l}$ while fixing $\left\{\mathbf{U}_{l}, \mathbf{W}_{l}, \boldsymbol{\Phi}_{l}\right\}$. By using Equation (1), we can obtain the optimal $\boldsymbol{\Psi}_{l}$ as:

$$
\mathbf{\Psi}_{l}=\mathbf{Z}_{l}\left(\mathbf{U}_{l},\left\{\mathbf{W}_{l}, \boldsymbol{\Phi}_{l}\right\}\right)^{-1} \text {. }
$$

3) Step 3: Solve (8) for $\mathbf{W}_{l}$ while fixing $\left\{\mathbf{U}_{l}, \boldsymbol{\Phi}_{l}, \mathbf{\Psi}_{l}\right\}$.

4) Step 4: Solve (8) for $\boldsymbol{\Phi}_{l}$ while fixing $\left\{\mathbf{U}_{l}, \mathbf{W}_{l}, \boldsymbol{\Psi}_{l}\right\}$.

Since there is no closed form solution in Steps 3 and 4, in the following we propose efficient methods to tackle Steps 3 and 4 .

\section{B. Optimization of The Precoding Matrices}

Here, we fix $\left\{\mathbf{U}_{l}, \boldsymbol{\Psi}_{l}, \boldsymbol{\Phi}_{l}\right\}$ and focus on the subproblem with respect to (w.r.t.) $\mathbf{W}_{l}$. Specifically, by substituting $\mathbf{E}_{l}$ into (8), and neglecting the irrelevant terms, the following problem can be formulated

$$
\min _{\mathbf{W}_{l}} \sum_{l} \operatorname{Tr}\left(\mathbf{W}_{l}^{H} \mathbf{A} \mathbf{W}_{l}\right)-2 \Re\left\{\operatorname{Tr}\left(\mathbf{B}_{l} \mathbf{W}_{l}\right)\right\}
$$

where $\mathbf{A}=\sum_{l} \omega_{l} \overline{\mathbf{H}}_{l}^{H} \mathbf{U}_{l} \mathbf{\Psi}_{l} \mathbf{U}_{l}^{H} \overline{\mathbf{H}}_{l}$, and $\mathbf{B}_{l}=\omega_{l} \mathbf{\Psi}_{l} \mathbf{U}_{l}^{H} \overline{\mathbf{H}}_{l}$, respectively.

(11) can be solved by a convex optimization solver such as CVX [14]. However, a more efficient method can be obtained by applying the duality. Specifically, the Lagrange cost function of (11) is

$$
\begin{aligned}
\mathcal{L}\left(\mathbf{W}_{l}, \lambda\right) & =\sum_{l} \operatorname{Tr}\left(\mathbf{W}_{l}^{H} \mathbf{A} \mathbf{W}_{l}\right)-2 \Re\left\{\operatorname{Tr}\left(\mathbf{B}_{l} \mathbf{W}_{l}\right)\right\} \\
& +\lambda\left(\sum_{l} \operatorname{Tr}\left(\mathbf{W}_{l} \mathbf{W}_{l}^{H}\right)-P_{s}\right) .
\end{aligned}
$$

where $\lambda>0$ is the dual variable w.r.t. (4b). By the first order optimization condition, we have $\mathbf{W}_{l}=(\mathbf{A}+\lambda \mathbf{I})^{-1} \mathbf{B}_{l}^{H}$. While $\lambda$ can be found by the bisection search method in [2], we omit the details for brevity.

\section{Optimization of The TRCs}

Here, we focus on optimizing $\boldsymbol{\Phi}_{l}$ for given $\left\{\mathbf{W}_{l}, \mathbf{U}_{l}, \mathbf{\Psi}_{l}\right\}$. Firstly, we substitute $\overline{\mathbf{H}}_{l}=\mathbf{H}_{l} \boldsymbol{\Phi}_{l} \mathbf{F} / \sigma_{l}$ into the objective of (11). Then, we have $\sum_{l} \operatorname{Tr}\left(\mathbf{W}_{l}^{H} \mathbf{A} \mathbf{W}_{l}\right)=$ $\sum_{l} \operatorname{Tr}\left(\boldsymbol{\Phi}_{l}^{H} \mathbf{H}_{l}^{H} \mathbf{U}_{l} \boldsymbol{\Psi}_{l} \mathbf{U}_{l}^{H} \mathbf{H}_{l} \boldsymbol{\Phi}_{l} \mathbf{F} \sum_{l} \omega_{l} \mathbf{W}_{l} \mathbf{W}_{l}^{H}\right) / \sigma_{l}^{2}$, and $\sum_{l} 2 \Re\left\{\operatorname{Tr}\left(\mathbf{B}_{l} \mathbf{W}_{l}\right)\right\}=\sum_{l} 2 \Re\left\{\operatorname{Tr}\left(\omega_{l} \mathbf{\Psi}_{l} \mathbf{U}_{l}^{H} \mathbf{H}_{l} \mathbf{\Phi}_{l} \mathbf{F} \mathbf{W}_{l}\right)\right\} / \sigma_{l}$.

Then, according to [15, Eq. (1.10.6)], for a diagonal matrix $\mathbf{E}=\operatorname{Diag}\left(e_{1}, \ldots, e_{m}\right) \in \mathbb{C}^{m \times m}$, we have $\operatorname{Tr}\left(\mathbf{E}^{H} \mathbf{C}_{1} \mathbf{E} \mathbf{C}_{2}\right)=\mathbf{e}^{H}\left(\mathbf{C}_{1} \odot \mathbf{C}_{2}^{T}\right) \mathbf{e}, \operatorname{Tr}\left(\mathbf{E} \mathbf{C}_{2}\right)=$ $\mathbf{1}^{T}\left(\mathbf{E} \odot \mathbf{C}_{2}^{T}\right) \mathbf{1}=\mathbf{e}^{T} \mathbf{c}_{2}$, and $\operatorname{Tr}\left(\mathbf{E}^{H} \mathbf{C}_{2}^{H}\right)=\mathbf{c}_{2}^{H} \mathbf{e}^{*}$, where $\mathbf{e}=\operatorname{diag}(\mathbf{E})$, and $\mathbf{c}_{2}=\operatorname{diag}\left(\mathbf{C}_{2}\right)$.

Thus, we obtain $\sum_{l} \operatorname{Tr}\left(\mathbf{W}_{l}^{H} \mathbf{A} \mathbf{W}_{l}\right)=\sum_{l} \phi_{l}^{H} \mathbf{Z}_{l} \phi_{l}$, and $\sum_{l} 2 \Re\left\{\operatorname{Tr}\left(\mathbf{B}_{l} \mathbf{W}_{l}\right)\right\}=\sum_{l} \phi_{l}^{H} \mathbf{z}_{l}^{*}$, respectively, where $\phi_{l}=$ $\operatorname{diag}\left(\boldsymbol{\Phi}_{l}\right)$, and

$$
\begin{array}{r}
\mathbf{Z}_{l}=\left(\omega_{l} \mathbf{H}_{l}^{H} \mathbf{U}_{l} \mathbf{\Psi}_{l} \mathbf{U}_{l}^{H} \mathbf{H}_{l}\right) \odot\left(\mathbf{F} \sum_{l} \mathbf{W}_{l} \mathbf{W}_{l}^{H} \mathbf{F}^{H}\right)^{T}, \quad \text { (13a) } \\
\mathbf{z}_{l}=\operatorname{diag}\left(\omega_{l} \mathbf{F} \mathbf{W}_{l} \mathbf{\Psi}_{l} \mathbf{U}_{l}^{H} \mathbf{H}_{l}\right) . \quad(13 \mathrm{~b})
\end{array}
$$

Based on above procedure, we formulate the following problem

$$
\begin{aligned}
& \min _{\phi_{l}} \sum_{l} \phi_{l}^{H} \mathbf{Z}_{l} \phi_{l} / \sigma_{l}^{2}-2 \Re\left\{\phi_{l}^{H} \mathbf{z}_{l}^{*}\right\} / \sigma_{l} \\
& \text { s.t. } \alpha_{m}^{t}+\alpha_{m}^{r}=1, \forall m \in \mathcal{M},
\end{aligned}
$$

The main difficulty in (14) is (14b), which is non-convex w.r.t to the amplitudes of the TRCs. To handle (14b), we define $\boldsymbol{\Omega}_{l} \triangleq \phi_{l}^{H} \phi_{l}, l \in\{t, r\}$. Then, it is easily shown that $\operatorname{diag}\left(\boldsymbol{\Omega}_{r}\right)=\left[\alpha_{1}^{r}, \ldots, \alpha_{M}^{r}\right]^{T}$ and $\operatorname{diag}\left(\boldsymbol{\Omega}_{t}\right)=\left[\alpha_{1}^{t}, \ldots, \alpha_{M}^{t}\right]^{T}$. Thus, (14b) is equivalent to $\operatorname{diag}\left(\boldsymbol{\Omega}_{r}+\boldsymbol{\Omega}_{t}\right)=\mathbf{1}$. Hence, we obtain the following problem

$$
\begin{aligned}
\min _{\phi_{l}, \boldsymbol{\Omega}_{l}} & \sum_{l} \phi_{l}^{H} \mathbf{Z}_{l} \phi_{l} / \sigma_{l}^{2}-2 \Re\left\{\phi_{l}^{H} \mathbf{z}_{l}^{*}\right\} / \sigma_{l} \\
\text { s.t. } & \phi_{r}^{H} \phi_{r}=\boldsymbol{\Omega}_{r}, \phi_{t}^{H} \phi_{t}=\boldsymbol{\Omega}_{t}, \\
& \operatorname{diag}\left(\boldsymbol{\Omega}_{r}+\boldsymbol{\Omega}_{t}\right)=\mathbf{1} .
\end{aligned}
$$

However, (15) is still non-convex due to (15b). The following Lemma is useful to handle (15b).

Lemma 2 [16]: The equality $\mathbf{T}=\mathbf{t t}^{H}$ is equivalent to

$$
\left\{\begin{array}{c}
{\left[\begin{array}{ccc}
\mathbf{D}_{1} & \mathbf{T} & \mathbf{t} \\
\mathbf{T}^{H} & \mathbf{D}_{2} & \mathbf{t} \\
\mathbf{t}^{H} & \mathbf{t}^{H} & 1
\end{array}\right] \succeq \mathbf{0}} \\
\operatorname{Tr}\left(\mathbf{t t}^{H}-\mathbf{D}_{1}\right) \geq 0,
\end{array}\right.
$$

where $\mathbf{D}_{1} \in \mathbb{H}^{N \times N}$ and $\mathbf{D}_{2} \in \mathbb{H}^{N \times N}$ are slack variables.

With Lemma 1, (15) can be recast as

$$
\begin{gathered}
\min _{\boldsymbol{\phi}_{l}, \boldsymbol{\Omega}_{l}} \sum_{l} \boldsymbol{\phi}_{l}^{H} \mathbf{Z}_{l} \phi_{l} / \sigma_{l}^{2}-2 \Re\left\{\phi_{l}^{H} \mathbf{z}_{l}^{*}\right\} / \sigma_{l} \\
\text { s.t. } \quad \operatorname{diag}\left(\boldsymbol{\Omega}_{r}+\boldsymbol{\Omega}_{t}\right)=\mathbf{1}, \\
{\left[\begin{array}{ccc}
\mathbf{D}_{1, l} & \boldsymbol{\Omega}_{l} & \phi_{l} \\
\boldsymbol{\Omega}_{l}^{H} & \mathbf{D}_{2, l} & \phi_{l} \\
\boldsymbol{\phi}_{l}^{H} & \boldsymbol{\phi}_{l}^{H} & 1
\end{array}\right] \succeq \mathbf{0},} \\
\operatorname{Tr}\left(\mathbf{D}_{1, l}-\boldsymbol{\phi}_{l} \boldsymbol{\phi}_{l}^{H}\right) \leq 0 .
\end{gathered}
$$


In fact, (17d) is non-convex, but can be approximated as

$$
\operatorname{Tr}\left(\mathbf{D}_{1, l}\right) \leq 2 \operatorname{Tr}\left(\boldsymbol{\phi}_{l}^{(n)} \boldsymbol{\phi}_{l}^{H}\right)-\operatorname{Tr}\left(\boldsymbol{\phi}_{l}^{(n)}\left(\boldsymbol{\phi}_{l}^{(n)}\right)^{H}\right)
$$

around given point $\phi_{l}^{(n)}$, where $n$ denotes the number of iterations. Thus, we formulate the following convex problem

$$
\begin{aligned}
& \min _{\phi_{l}, \boldsymbol{\Omega}_{l}} \sum_{l} \phi_{l}^{H} \mathbf{Z}_{l} \phi_{l} / \sigma_{l}^{2}-2 \Re\left\{\phi_{l}^{H} \mathbf{z}_{l}^{*}\right\} / \sigma_{l} \\
& \text { s.t. }(17 \mathrm{~b}),(17 \mathrm{c}), \\
& \operatorname{Tr}\left(\mathbf{D}_{1, l}\right) \leq 2 \operatorname{Tr}\left(\phi_{l}^{(n)} \phi_{l}^{H}\right)-\operatorname{Tr}\left(\phi_{l}^{(n)}\left(\phi_{l}^{(n)}\right)^{H}\right),
\end{aligned}
$$

which can be solved by a convex optimization solver such as CVX [14]. ${ }^{2}$

\section{Convergence and Computational Complexity analysis}

Here, we provide a brief proof for the convergence of the BCD method. Firstly, according to [17], the convergence of the inner CCCP method can be guaranteed. As for the outer BCD algorithm, we denote the objective value sequence of (4) as $\left\{R\left(\mathbf{W}_{l}^{k}, \boldsymbol{\Phi}_{l}^{k}\right)\right\}$, and the objective function of (8) as $f\left(\mathbf{W}_{l}, \boldsymbol{\Phi}_{l}, \mathbf{U}_{l}, \mathbf{\Psi}_{l}\right)$. Then, we have

$$
\begin{aligned}
& R\left(\mathbf{W}_{l}^{k+1}, \boldsymbol{\Phi}_{l}^{k+1}\right)=f\left(\mathbf{W}_{l}^{k+1}, \boldsymbol{\Phi}_{l}^{k+1}, \mathbf{\Psi}_{l}^{k+1}, \mathbf{U}_{l}^{k+1}\right) \\
& \quad \geq f\left(\mathbf{W}_{l}^{k+1}, \boldsymbol{\Phi}_{l}^{k+1}, \mathbf{\Psi}_{l}^{k+1}, \mathbf{U}_{l}^{k}\right) \\
& \quad \geq f\left(\mathbf{W}_{l}^{k+1}, \boldsymbol{\Phi}_{l}^{k+1}, \mathbf{\Psi}_{l}^{k}, \mathbf{U}_{l}^{k}\right) \\
& \quad \geq f\left(\mathbf{W}_{l}^{k}, \boldsymbol{\Phi}_{l}^{k+1}, \mathbf{\Psi}_{l}^{k}, \mathbf{U}_{l}^{k}\right) \\
& \quad \geq f\left(\mathbf{W}_{l}^{k}, \boldsymbol{\Phi}_{l}^{k}, \mathbf{\Psi}_{l}^{k}, \mathbf{U}_{l}^{k}\right)=R\left(\mathbf{W}_{l}^{k}, \boldsymbol{\Phi}_{l}^{k}\right) .
\end{aligned}
$$

Since $R\left(\mathbf{W}_{l}^{k}, \boldsymbol{\Phi}_{l}^{k}\right)$ is upper bounded due to (4b), (20) generates a monotonic sequence $\left\{R\left(\mathbf{W}_{l}^{k}, \boldsymbol{\Phi}_{l}^{k}\right)\right\}$, thus is guaranteed to converge. More details are in [13].

The overall BCD algorithm is given in Algorithm 1.

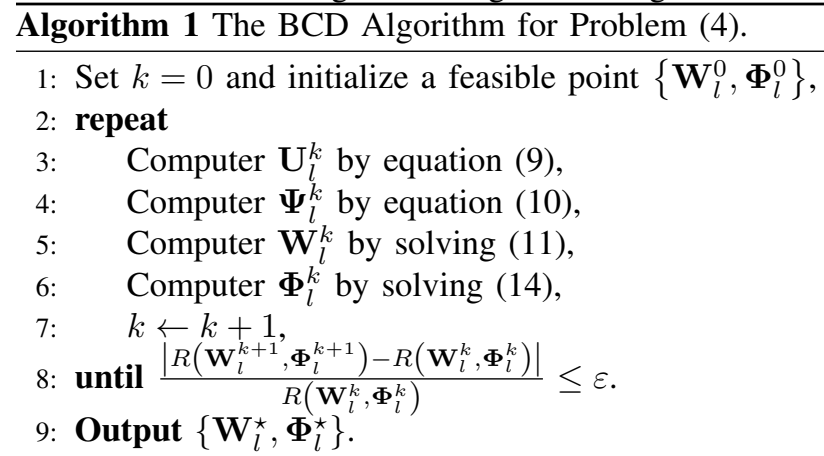

Now, we estimate the computational complexity of the BCD algorithm, which is mainly determined by the complexity of Steps 3 and 4 in Algorithm 1. Specifically, for the optimization of $\mathbf{W}_{l}$, the main complexity is to calculate $\mathbf{W}_{l}$ in (11), which is given by $O\left(N_{d_{t}}^{3} N^{3}+N_{d_{r}}^{3} N^{3}\right)$, and the required number of iterations is $\log _{2}\left(\frac{\lambda_{u}-\lambda_{l}}{\varepsilon}\right)$, where $\lambda_{u}$ and $\lambda_{l}$ denote the upper and lower bounds on $\lambda$, and $\varepsilon$ denotes the required accuracy. Hence, the total complexity of (11) is $O\left(\log _{2}\left(\frac{\lambda_{u}-\lambda_{l}}{\varepsilon}\right)\left(N_{d_{t}}^{3}+N_{d_{r}}^{3}\right) N^{3}\right)$ [3].

Then, for the CCCP method to update $\boldsymbol{\Phi}_{l}$, according to [?], in each iteration, the complexity of solving (19) is $O\left(M^{3}\right)$. Thus, the total complexity is $O\left(T M^{3}\right)$, where $T$ denotes the

\footnotetext{
${ }^{2}$ For the broadcast scenario with the ES method, the Tx employs one precoding matrix $\mathbf{W} \in \mathbb{C}^{N \times N_{d}}$ to convey the same symbol. Then, by defining $\mathbf{U}_{l}=\left(\overline{\mathbf{H}}_{l} \mathbf{W} \mathbf{W}^{H} \overline{\mathbf{H}}_{l}^{H}+\sigma_{l}^{2} \mathbf{I}\right)^{-1} \overline{\mathbf{H}}_{l} \mathbf{W}, \quad \mathbf{\Psi}_{l}=$ $\mathbf{E}_{l}\left(\mathbf{U}_{l}, \mathbf{W}\right)^{-1}, \mathbf{Z}_{l}=\left(w_{l} \mathbf{H}_{l}^{H} \mathbf{U}_{l} \mathbf{\Psi}_{l} \mathbf{U}_{l}^{H} \mathbf{H}_{l}\right) \odot\left(\mathbf{F W} \mathbf{W}^{H} \mathbf{F}^{H}\right)^{T}$, and $\mathbf{z}_{l}=\operatorname{diag}\left(w_{l} \mathbf{F} \mathbf{W} \boldsymbol{\Psi}_{l} \mathbf{U}_{l}^{H} \mathbf{H}_{l}\right)$, respectively, we can obtain the corresponding method for broadcast communication with the ES scheme.
}

number of iterations. According to the above analysis, the overall complexity of the BCD method can be estimated by $C=O\left(\max \left\{\log _{2}\left(\frac{\lambda_{u}-\lambda_{l}}{\varepsilon}\right)\left(N_{d_{r}}^{3}+N_{d_{l}}^{3}\right) N^{3}, T M^{3}\right\}\right)$.

From (21), we can see that polynomial time complexity can be obtained by the proposed algorithm, making it suitable for practical implementation.

\section{Simulation Results}

Fig. 1 illustrates the considered simulation scenario, where Tx and the STAR-RIS are located at $(0,0,10)$ and $(0,30,10)$ meters, respectively. The users are randomly deployed on half-circles centered at the RIS, with radius of $5 \mathrm{~m}$, and heights of $2 \mathrm{~m}$. In addition, we set $N=4$, $P_{s}=30 \mathrm{dBm}, M=30, N_{l}=4, \sigma_{l}^{2}=-80 \mathrm{dBm}$, $\omega_{l}=0.5, \forall l$. The path loss is given by $\mathrm{PL}=\mathrm{PL}_{0}\left(d / d_{0}\right)^{-\beta}$, where $\mathrm{PL}_{0}=10^{-3}$ is the channel gain at $d_{0}=1 \mathrm{~m}, d$ is the link distance, and $\beta=2.2$ is the link loss exponent. While the small scale fading channels $\mathbf{F}$ is modeled as $\mathbf{F}=\sqrt{\frac{K_{T R}}{K_{T R}+1}} \mathbf{F}^{\mathrm{LoS}}+\sqrt{\frac{1}{K_{T R}+1}} \mathbf{F}^{\mathrm{NLoS}}$, with the Rician factor $K_{T R}=5 \mathrm{~dB}$. In addition, $\mathbf{F}^{\mathrm{LoS}}=\mathbf{a b}^{H}$ represents the line-of-sight $(\mathrm{LoS})$ component, with $\mathbf{a}=\left[a_{1}, \ldots, a_{N}\right]^{T}$ and $\mathbf{b}=\left[b_{1}, \ldots, b_{M}\right]^{T}$ denote the steering vectors, while $\mathbf{F}^{\mathrm{NLoS}}$ is the non-LoS component and modeled as the Rayleigh fading. The rest channels can be obtained in a similar way.

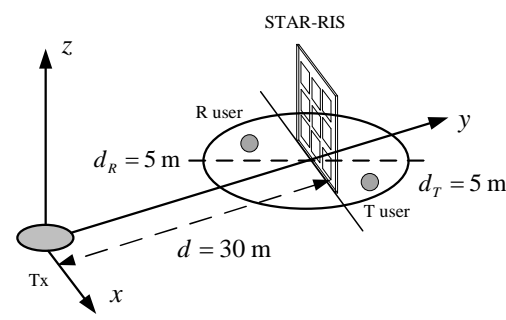

Fig. 1. The investigated scenario of the STAR-RIS-aided network.

Firstly, we analyze the convergence of the proposed method. Fig. 2 plots the WSR versus the number of iterations. From this figure, we can see that the WSR tends to converge to a stable value in almost 10 iterations. In addition, larger $N, M$ or $N_{l}$ achieves a higher WSR at the cost of slower convergence speed, since more variables need to be optimized.

Then, we compare the proposed design with some benchmarks: 1) The equal ES scheme, e.g., fixing $\alpha_{m}^{t}=\alpha_{m}^{r}=$ $0.5, \forall m \in \mathcal{M}$ while only optimizing $\phi_{m}^{t}$ and $\left.\phi_{m}^{r} ; 2\right)$ The conventional RISs method, where one $M / 2$ elements reflectingonly RIS and one $M / 2$ elements transmitting-only RIS are deployed at the same position as the STAR-RIS, and adjacent to each other. This configuration can provide a fair comparison between STAR-RIS and conventional RISs; 3) The SDR method with ES scheme in [11]. These approaches are labelled as "ES scheme", "TS scheme", "MS scheme", "Equal ES", "Conventional RIS", and "SDR method", respectively.

Firstly, we show the WSR versus the transmit power budget $P_{s}$ in Fig. 3, in the unicast communication scenario. From Fig. 3, we can see that the WSR increases with $P_{s}$ for all these schemes, while the TS scheme achieves higher WSR than other methods, especially in the high $P_{s}$ region, since it can eliminate the inter-user interference. Besides, STARRIS achieves better performance than conventional RISs, since conventional RISs use given numbers of elements for the two modes, thus the degrees-of-freedom (DoFs) can not be 


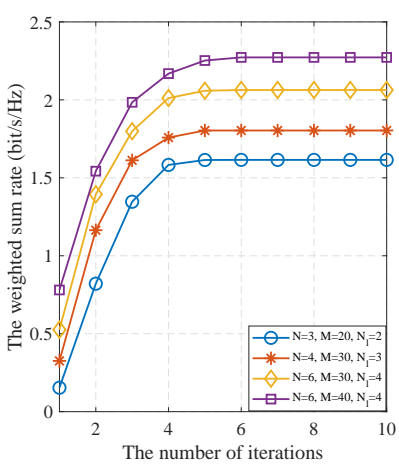

Fig. 2. The WSR versus the number Fig. 3. The WSR versus $P_{s}$ in unicast of iterations.

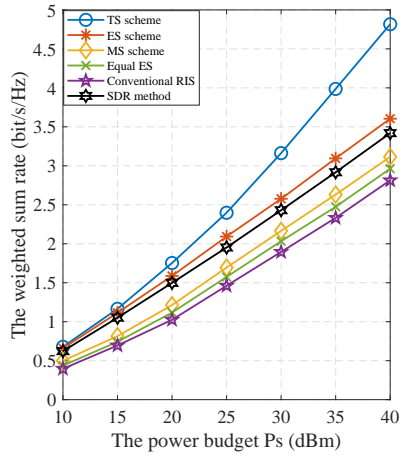

communication.

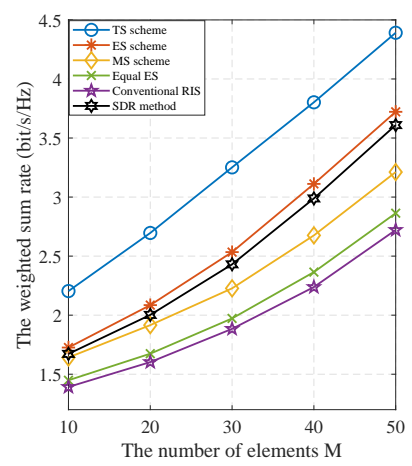

Fig. 4. The WSR versus $M$ in unicast Fig. 5. The WSR versus $P_{s}$ in communication.

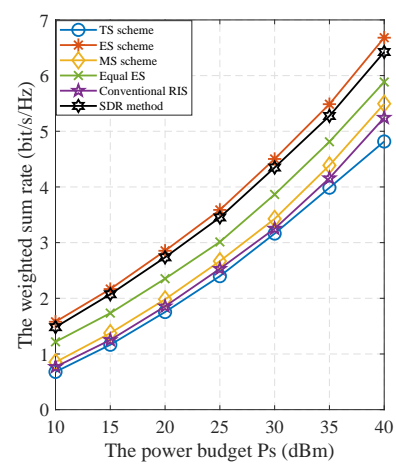

broadcast communication fully exploited. While the SDR method results in certain performance loss, since the relaxation of the rank constraint may impair the structure of the solution.

Next, we show the WSR versus the number of RIS elements $M$ in Fig. 4, in the unicast communication scenario. Here, we can find that the WSR increases with $M$ for all these schemes, since with larger $M$, more signals can arrive at the STAR-RIS. And with properly optimized TRCs, the sum of the transmitting and reflecting signals increases, thus to improve the WSR.

Lastly, we plot the WSR versus $P_{s}$ in Fig. 5, in the broadcast communication scenario. Different from the results in Fig. 3, in this case, the ES and MS schemes achieve better performance than the TS scheme. This is mainly due to the fact that in the broadcast communication scenario, there is no inter-user interference for the ES or MS scheme, and the two schemes can utilize the total available communication time to improve the WSR.

\section{CONCLUSION}

This work investigated the synergy between the $\mathrm{T}$ and $\mathrm{R}$ modes for the STAR-RIS-enhanced MIMO downlink channel. The WSR objective was solved the with BCD and CCCP methods for the ES scheme. Simulation results verified the performance of the proposed method as well as the superiority of the STAR-RIS technique.

\section{REFERENCES}

[1] Y. Liu, X. Liu, X. Mu, T. Hou, J. Xu, Z. Qin, M. D. Renzo, and N. Al-Dhahir, "Reconfigurable intelligent surfaces: Principles and opportunities," IEEE Commun. Surveys Tut., vol. 23, no. 3, pp. 1546-1577, 3rd Quart. 2021.

[2] C. Pan, H. Ren, K. Wang, W. Xu, M. Elkashlan, A. Nallanathan, and L. Hanzo, "Multicell MIMO communications relying on intelligent reflecting surface," IEEE Trans. Wireless Commun., vol. 19, no. 8, pp. 5218-5233, Aug. 2020.

[3] C. Pan, H. Ren, K. Wang, M. Elkashlan, A. Nallanathan, J. Wang, and L. Hanzo, "Intelligent reflecting surface aided MIMO broadcasting for simultaneous wireless information and power transfer," IEEE J. Sel. Areas Commun., vol. 38, no. 8, pp. 1719-1734, Aug. 2020.

[4] X. Mu, Y. Liu, L. Guo, J. Lin, and H. V. Poor, "Intelligent reflecting surface enhanced multi-UAV NOMA networks," IEEE J. Sel. Areas Commun., vol. 39, no. 10, pp. 3051-3066, Oct. 2021.

[5] S. Zeng, H. Zhang, B. Di, Y. Tan, Z. Han, H. V. Poor, and L. Song, "Reconfigurable intelligent surfaces in 6G: reflective, transmissive, or both?" IEEE Commun. Lett., vol. 25, no. 6, pp. 2063-2067, Jun. 2021.

[6] H. Zhang, S. Zeng, B. Di, Y. Tan, M. D. Renzo, M. Debbah, L. Song, Z. Han, and H. V. Poor, "Intelligent reflective-transmissive metasurfaces for full-dimensional communications: Principles, technologies, and implementation," arXiv preprint arXiv:2104.12313v1, 2021.
[7] S. Zhang, H. Zhang, B. Di, Y. Tan, M. D. Renzo, Z. Han, H. V. Poor, and L. Song, "Intelligent omni-surface: Ubiquitous wireless transmission by reflective-transmissive metasurfaces," arXiv preprint arXiv:2011.00765v1, 2020.

[8] S. Zhang, H. Zhang, B. Di, Y. Tan, Z. Han, and L. Song, "Beyond intelligent reflecting surfaces: reflective-transmissive metasurface aided communications for full-dimensional coverage extension," IEEE Trans. Veh. Tech., vol. 69, no. 11, pp. 13905-13909, Nov. 2020.

[9] J. Xu, Y. Liu, X. Mu, and O. A. Dobre, "STAR-RISs: Simultaneous transmitting and reflecting reconfigurable intelligent surfaces," IEEE Commun. Lett., vol. 25, no. 9, pp. 3134-3138, Sep. 2021.

[10] J. Xu, Y. Liu, X. Mu, J. T. Zhou, L. Song, H. V. Poor, and L. Hanzo, "Simultaneously transmitting and reflecting (STAR) intelligent omni-surfaces, their modeling and implementation," arXiv preprint arXiv:2108.06233v1, 2021.

[11] X. Mu, Y. Liu, L. Guo, J. Lin, and R. Schober, "Simultaneously transmitting and reflecting (STAR) RIS aided wireless communications," IEEE Trans. Wireless Commun., doi: 10.1109/TWC.2021.3118225.

[12] H. Niu, Z. Chu, F. Zhou, and Z. Zhu, "Simultaneous transmission and reflection reconfigurable intelligent surface assisted secrecy MISO networks," IEEE Commun. Lett., vol. 25, no. 11, pp. 3498-3502, Nov. 2021.

[13] Q.-J. Shi, W.-Q. Xu, J.-S. Wu, E.-B. Song, and Y.-M. Wang, "Secure beamforming for MIMO broadcasting with wireless information and power transfer," IEEE Trans. Wireless Commun., vol. 14, no. 5, pp. 28412853, May 2015.

[14] M. Grant and S. Boyd, CVX: Matlab software for disciplined convex programming, version 2.0 beta, 2012. [online]. Available: http://cvxr.com/cvx.

[15] X.-D. Zhang, Matrix analysis and applications. Cambridge University Press, 2017.

[16] U. Rashid, H. D. Tuan, H. H. Kha, and H. H. Nguyen, "Joint optimization of source precoding and relay beamforming in wireless MIMO relay networks," IEEE Trans. Commun., vol. 62, no. 2, pp. 488-499, Feb. 2014.

[17] A. J. Smola, S. V. N. Vishwanathan, and T. Hofmann, "Kernel methods for missing variables," in Proc. 10th Int. Workshop Artif. Intell. Statist., Mar. 2005, pp. 1-8.

[18] Z. Lin, M. Lin, T. de Cola, J.-B. Wang, W.-P. Zhu, and J. Cheng, "Supporting IoT with rate-splitting multiple access in satellite and aerial integrated networks," IEEE Internet of Things J., vol. 8, no. 14, pp. 11123-11134, Jul. 2021. 